\title{
2013 Scientific Referees
}

(C) Italian Society of Orthopaedics and Traumatology 2014

The quality of the Journal of Orthopaedics and Traumatology depends on the qualified and regular collaboration of renowned scientists who devoted their time to

Roberto Adani (Verona, Italy)

Ezio Adriani (Rome, Italy)

Gabriele Agati (Orbassano, Italy)

Mustafa Alnaib (Gateshead, UK)

Antonio Andreacchio (Turin, Italy)

Alessandro Aprato (Turin, Italy)

Stefano Artiaco (Turin, Italy)

Stefano Astolfi (Rome, Italy)

Andrea Atzei (Verona, Italy)

Francesco Atzori (Turin, Italy)

Angelo Aulisa (Rome, Italy)

Pavan Kumar Avadhanam (Karimnagar, India)

Mostafa Ayoub (Tanta, Egypt)

Brodano Giovanni Barbanti (Bologna, Italy)

Vittorio Bellotti (Barcelone, Spain)

Teresa Benigno (Turin, Italy)

Federica Bergamin (Turin, Italy)

Alexander Berth (Magdeburg, Germany)

Celeste Bertone (San Donato Milanese, Italy)

Giuseppe Bianchi (Bologna, Italy)

Amin Bigham (Shahrekord, Islamic Republic of Iran)

Amedeo Bini (Varese, Italy)

Kristian Bjorgul (Fredrikstad, Norway)

Silvio Luigi Boero (Genoa, Italy)

Andrea Borgo (Padua, Italy)

Mario Borroni (Rozzano, Italy)

Roberto E. Buda (Bologna, Italy)

Matteo Cadossi (Bologna, Italy)

Filippo Calderazzi (Parma, Italy)

Domenico Campanacci (Florence, Italy)

Laura Campanacci (Bologna, Italy)

Fabrizio Campi (Cattolica, Italy)

Christian Carulli (Florence, Italy)

Maurizio Catagni (Lecco, Italy) constructively review the submitted articles. We are indebted to the following experts who reviewed papers which completed the peer-reviewing process within 2013.

Simone Cerciello (Rome, Italy)

Riccardo Ciatti (Rome, Italy)

Claudio Cimminiello (Vimercate, Italy)

Norberto Confalonieri (Milan, Italy)

Luigi Conforti (Turin, Italy)

Aurélien Courvoisier (Grenoble, France)

Marco Crostelli (Rome, Italy)

Walter Daghino (Turin, Italy)

Carlo Dall'Oca (Verona, Italy)

Umberto De Bellis (Milan, Italy)

Orazio De Lucia (Milan, Italy)

Luigi De Palma (Ancona, Italy)

Marco De Peppo (Rome, Italy)

Ferdinando Del Prete (Florence, Italy)

Giacomo Delle Rose (Rozzano, Italy)

Angelo Dettoni (Turin, Italy)

Shabir A. Dhar (Srinagar, India)

Giovanni L. Di Gennaro (Bologna, Italy)

Alberto Di Martino (Rome, Italy)

Vitantonio Digennaro (Bologna, Italy)

Francesco Falciglia (Rome, Italy)

Pasquale Farsetti (Rome, Italy)

Francesco Fauci (Cattolica, Italy)

Flavio Fazioli (Naples, Italy)

Andrea Ferretti (Rome, Italy)

Florian Fischer (Milan, Italy)

Maurizio Fontana (Faenza, Italy)

Matteo Formica (Genoa, Italy)

Olimpio Galasso (Catanzaro, Italy)

Eduardo García-Rey (Madrid, Spain)

Alessandro Gasbarrini (Bologna, Italy)

Giorgio Gasparini (Catanzaro, Italy)

Matthew C. Giordano (Rome, Italy)

Enrico Guerra (Bologna, Italy) 
Tetsuo Hagino (Kofu, Japan)

Massimo Innocenti (Florence, Italy)

Ernesto Ippolito (Rome, Italy)

Lukas Iselin (Basel, Switzerland)

Nicola Ivaldo (Acqui Terme, Italy)

Ismail Emre Ketenci (Istanbul, Turkey)

Yousuf Khira (Zagazig, Egypt)

Ozkan Kose (Antalya, Turkey)

Antonios Kouzelis (Patras, Greece)

Arun Kumar (Manchester, UK)

Danilo Leonetti (Bologna, Italy)

Giandomenico Logroscino (Rome, Italy)

Giovanni Lovisetti (Menaggio, Italy)

Bruno Magnan (Verona, Italy)

Devendra Mahadevan (Leicester, UK)

Alessio Maiello (Turin, USA)

Fabrizio Margheritini (Roma, Italy)

Matteo Marullo (Pavia, Italy)

Marcos Matos (Salvador, Brazil)

Cyril Mauffrey (Denver, USA)

Barbara Melis (Cagliari, Italy)

Giovanni Merolla (Cattolica, Italy)

Jane Christiane Messina (Milan, Italy)

Giovanni Monteleone (Rome, Italy)

George Mouzopoulos (Athens, Greece)

Thomasz Nizegorodcew (Rome, Italy)

Angela Notarnicola (Bari, Italy)

Masahiko Nozawa (Nerima-ku, Japan)

Matthew Oetgen (New Haven, USA)

Alessia Pagnotta (Roma, Italy)

Paolo Paladini (Cattolica, Italy)

Matteo Palmisani (Modena, Italy)

Bernardino Panero (Turin, Italy)

Hemant Parekh (Pune, India)

Vinay Parmar (Manchester, UK)

Shelain Patel (London, UK)

Vito Pavone (Catania, Italy)

Alessio Pedrazzini (Parma, Italy)

Giovanni Peretti (Milan, Italy)

Giuseppe Peretti (Milan, Italy)

Franco Pleitavino (Genoa, Italy)

Enrico Pola (Rome, Italy)
Domenico Prisco (Florence, Italy)

Michele Rampoldi (Rome, Italy)

Enrico Rebuzzi (Oderzo, Italy)

Dario Regis (Verona, Italy)

Olav Reikerås (Oslo, Norway)

Stefano Rigotti (Negrar, Italy)

Giovanni Risitano (Messina, Italy)

E. Carlos Rodriguez-Merchan (Madrid, Spain)

Carlo L. Romanò (Milan, Italy)

Stefano M.P. Rossi (Pavia, Italy)

Claudio Rovesta (Modena, Italy)

Raffaele Russo (Naples, Italy)

Luigi Sabatini (Saluzzo, Italy)

Kabul Saikia (Guwahati, India)

Nicola Santori (Milan, Italy)

Alfredo Schiavone Panni (Campobasso, Italy)

Vincenzo Sessa (Rome, Italy)

Hamid Reza Shemshaki (Isfahan, Islamic Republic of Iran)

Ahmed Siam (Alexandria, Egypt)

Saurabh Singh (Varanasi, India)

Ashutosh Singh (Lucknow, India)

Bhagwat Singh Rao (Ahmedabad, India)

Giuseppe Solarino (Bari, Italy)

Luigi Tarallo (Modena, Italy)

Saket Tibrewal (London, UK)

Davut Tiren (Breda, Netherlands)

Gianpaolo Torasso (Orbassano, Italy)

Pierluigi Tos (Turin, Italy)

Federico Trentani (Bologna, Italy)

Paolo Trentani (Bologna, Italy)

Cosimo Tudisco (Rome, Italy)

Gianluca Vadalà (Rome, Italy)

Luca Vaienti (San Donato Milanese, Italy)

Francesca Vannini (Bologna, Italy)

Michele Arcangelo Verdano (Parma, Italy)

Paulo Roberto Vilaça Júnior (São Paulo, Brazil)

Anne Vochteloo (Leiden, Netherlands)

Istemi Yucel (Düzce, Turkey)

Stefano Zaffagnini (Bologna, Italy)

Gustavo Zanoli ( Ferrara, Italy)

Giovanni Zatti (Monza, Italy) 